\title{
Ein Blick zurück in die Gründungszeit der Gutachterstelle
}

\author{
Hans Ott, Fürsprecher, ehem. Generalsekretär der FMH
}

Nachdem in den 70er Jahren die «hohen ÄrzteEinkommen» im Schussfeld der Boulevardpresse gewesen waren, kamen Anfang der 80er Jahre die ärztlichen Kunstfehler unter Beschuss. Wenn ich mich recht erinnere, führte der «Blick» damals gar eine ständige Rubrik dafür und forderte die Leser auf, sie zu alimentieren. In parlamentarischen Vorstössen wurde eine Kausalhaftung für Ärzte gefordert, d. h. eine Haftung des Arztes für jeden Schaden aus seiner Berufsausübung, unbekümmert darum, ob ihm ein Behandlungsfehler unterlaufen sei oder nicht.

All das veranlasste mich, im Oktober 1980 zu einem Seminar der Kadermitarbeiter und der Sekretäre der kantonalen Ärztegesellschaften auch prominente Vertreter der schweizerischen Haftpflichtversicherer einzuladen. Man wurde sich bald einig darüber, dass die Behandlung von Ärztehaftpflichtfällen im Rahmen des geltenden Systems rasch verbessert werden müsse, wenn eine Systemveränderung vermieden werden solle. Es galt, den Patienten, aber letztlich auch den betroffenen Ärzten, eine Institution zur Verfügung zu stellen, die es ermöglichen sollte, ohne teure Gerichtskosten die Behandlungsfehlerfrage abklären zu können.

Die Ausgangssituation für Versicherer und FMH war damals insofern günstig, als Fürsprecher K. Tännler, Direktor der «Vaudoise», Präsident der Vereinigung der Haftpflicht- und Motorfahrzeugversicherer (HMV) war. K. Tännler war ein Studienkollege von mir und Auditor am Div Ger 10 B, das ich präsidierte. Dieses günstige Verhandlungsklima führte binnen zweier Monaten zu einem Entwurf für ein «Reglement für die aussergerichtliche Begutachtung von Ärztehaftpflichtfällen», dessen Kern eine vom Generalsekretariat zu führende «Gutachterstelle für den Fall behaupteter ärztlicher Behandlungs- und Diagnosefehler» sein sollte.

Hauptzweck dieser Gutachterstelle war die Vermittlung qualifizierter Gutachter an Patienten, die sich als Opfer ärztlicher Kunstfehler sahen - was mit Hilfe der medizinischen Fachgesellschaften geschehen sollte. Durch eine qualifizierte «Betreuung» des Begutachtungsverfahrens sollte auch bewirkt werden, dass die Gutachten binnen vernünftiger Frist erstattet würden. Und, last but not least, die Frage, ob ein - meist kostspieliges - Gutachten eingeholt werden solle oder nicht, sollte dadurch entschärft werden, dass die Gutachterstelle die Kosten übernehmen würde. Intern war der «Verteiler» der, dass sowohl die FMH wie auch die HMV an die Kosten der neuen Institution beitragen würden. Bewusst sah der Reglemententwurf davon ab, zwischen dem geschädigten Patienten und dem (oder den) Verantwortlichen auch zu schlichten und damit insbesondere über die Schadenersatzansprüche selbst entscheiden zu wollen. In diesem Punkt wich der Reglemententwurf von den Reglementen anderer Länder ab.

Das verhältnismässig einfache Konzept vermochte im Laufe des Jahres 1981 den FMHZentralvorstand, die Präsidentenkonferenz und auch die HMV zu überzeugen, so dass die Gutachterstelle bereits im Februar 1982 ihre Arbeit aufnehmen konnte. Aber wohlverstanden: das Reglement sah weder eine Verpflichtung der Fachgesellschaften (zur Vermittlung der Gutachter) noch eine Einlassungspflicht der (eines Kunstfehlers bezichtigten) Ärzte vor; das System beruhte auf Freiwilligkeit, von der Auferlegung von Pflichten wurde bewusst abgesehen, um die Gutachterstelle rasch in Betrieb nehmen zu können. Erst rund 10 Jahre später hat die Ärztekammer durch einen sogenannten allgemeinverbindlichen Beschluss die Einlassungspflicht des betroffenen Arztes eingeführt.

Im ersten Jahresbericht der Gutachterstelle konnte ich feststellen, dass sich das Konzept trotz einiger Pannen bewährt hatte [1]. Auch das Misstrauen des Publikums und der Patientenorganisationen («Persilstellen?»), aber auch der Ärzte, begann sich zu zerstreuen. Dazu beigetragen hatte auch die ganz beiläufig aufgekommene Idee, die Gutachterstellen extern führen zu lassen durch Frau Susanne Friedli in der deutschsprachigen Schweiz, Frau Brigitte Mottet in der Romandie. Die zwei Frauen wirkten - und wirken - ausgesprochen vertrauensbildend.

Und nun ist die Gutachterstelle schon 20 Jahre alt. Ihr Reglement ist mehrfach revidiert und zusammen mit dem Kommentar, den Anleitungen und Musterbriefen zu einem regelrechten Regelwerk geworden. Soweit ich das als 
- seit 15 Jahren - Aussenstehender beurteilen kann, geniesst sie das Vertrauen aller Beteiligten und Betroffenen. Die ärztliche Haftpflicht«szene» ist heute entspannt, die Boulevardpresse hat sich, die jüngste Vergangenheit zeigt es, anderen Zielgruppen zugewandt. Die Ärzteschaft wird weniger schlecht gemacht als auch schon.

Die neugeschaffene Meldestelle für Komplikationen und Fehler (CIRS-Medical) wird ihrerseits dazu beitragen, dass aus festgestellten Fehlern und Komplikationen allgemein gelernt werden kann. Das scheint mir umso wichtiger, als der vorliegende Entwurf für die Haftpflicht- normen des OR für besonders gefährliche Tätigkeiten eine Gefährdungshaftung vorsieht, womit die Haftpflicht der Ärzte - welche üben eine «besonders gefährliche» Tätigkeit aus? - erneut ins Blickfeld der Öffentlichkeit geraten wird. Mit der Ruhe wird es also bereits in absehbarer Zeit aus sein.

\section{Literatur}

1 Ott H. Rückblick auf ein Jahr Gutachterstelle für Ärztehaftpflichtfälle. Schweiz Ärztezeitung 1983;64(31):1159-60.

\section{Premiers pas du Bureau d'expertises extrajudiciaires en Suisse romande}

François-Xavier Deschenaux, ancien Secrétaire général de la FMH

\section{Une demande de changement de la part du public}

Les conditions et l'environnement socio-politique ayant présidé à la mise sur pied du Bureau d'expertises extrajudiciaires de la FMH (ci-après: B.Exp.) ont été fondamentalement les mêmes des deux côtés de la Sarine. Ici aussi la pression se fit grande dans certains milieux de l'opinion pour que l'on quitte la responsabilité uniquement basée sur la faute pour liquider les contentieux ayant pour objet les dommages d'origine iatrogène. En particulier, le cas spectaculaire et abondamment relaté dans la presse romande écrite et parlée d'un malheureux patient dont la vie quotidienne et économique avait été bouleversée (défiguration irréversible, dommages financiers très élevés) suite à une hyperréaction allergique que rien ne permettait de prévoir à un produit à base de iode utilisé comme produit de contraste n'avait pas peu contribué à gagner des partisans résolus en faveur d'un système de responsabilité purement causale, indépendante de la présence ou de l'absence d'une faute person-
Il était donc grand temps, pour contrecarrer ces efforts insistants préconisant un abandon du système de responsabilité en vigueur, d'assurer aux patients ou à leur ayants-cause des conditions ne faisant pas de la recherche d'un médecin expert à qui confier de légitimes questions sur l'existence ou non d'une faute médicale, un véritable parcours du combattant, souvent long, coûteux et pour finir décourageant.

Les premiers pas du B.Exp. furent à certains égards favorisés par le fait que son responsable juriste pour la Suisse romande était par ailleurs également en charge du Service romand d'information médicale (SRIM), ces deux antennes de la FMH étant toutes deux réunies dans les mêmes locaux à Lausanne. Outre que cela constituait un poste d'observation privilégié pour percevoir les attentes du public et des journalistes et dispenser sans délais les informations sollicitées, cela permit également grâce à une synergie fort efficace, de corriger rapidement certains malentendus et de faire bonne justice de certains procès d'intention, peut-être inévitables à l'origine.

Preuve que la mise sur pied de ce B.Exp. correspondait bien à un réel besoin, on dût consta- 
ter dans les premières années que chaque information dispensée sur le sujet ou chaque interview accordée à la presse sur le fonctionnement de ce bureau entraînait des retombées immédiates dans les semaines qui suivaient sur le nombre de procédures introduites devant ce bureau, au point que le reproche fut parfois formulé que le SRIM animait artificiellement la demande du public.

\section{Une confiance fondée sur l'expérience}

Parmi les résistances psychologiques à vaincre du côté du public et plus encore du côté de certains journalistes, le fait que des médecins rendaient des expertises sur les activités de leurs confrères et consœurs fut celui qui rencontra au début le plus de scepticisme. Il fallut faire œuvre de persuasion pour convaincre les critiques que l'on ne pouvait guère confier des expertises à d'autres professionnels que les médecins et qu'il était préférable que ces derniers fussent des médecins de ce pays sur lesquels l'organisation professionnelle pouvait exercer son influence, que ce soit pour présider au choix des experts, surveiller le respect des délais et modérer au besoin les honoraires.
Les résultats statistiques des premiers exercices annuels de l'activité du B.Exp. contribuèrent grandement à convaincre les plus critiques que cette institution n'avait rien d'une entreprise générale de blanchissage des médecins que d'aucuns redoutaient. Le taux des fautes professionnelles retenues par les experts prouva à satisfaction que les experts commis par le B.Exp. ne concevaient nullement leur mission comme consistant à jeter le manteau de Noé sur les erreurs de leurs confrères et consœurs, que l'on ne pouvait pas leur faire le grief d'avoir une approche corporatiste de leur mandat et que les patients disposaient donc d'une réelle chance d'obtenir satisfaction.

Le fait qu'assez rapidement des administrateurs d'hôpitaux publics romands aient recherché spontanément le concours d'une juridiction privée telle que celle offerte par le B.Exp. put être considérée comme un succès d'estime pour les activités de ce bureau. Cela contribua aussi certainement à vaincre des résistances émanant du milieu médical lui-même à l'encontre de cette institution. La décision de la Chambre médicale acquise à une très large majorité rendant la juridiction de ce bureau obligatoire pour tous les membres de la FMH acheva d'asseoir son autorité au sein de la profession. 